\title{
EDITORIAL
}

\section{FORMACIÓN, LIDERAZGO Y UN CASO REAL DE LÍDER TRANSFORMACIONAL EN LA UNIVERSIDAD}

\author{
TEACHING, LEADERSHIP AND A CASE OF TRANSFORMATIONAL \\ LEADERSHIP STYLE IN THE UNIVERSITY
}

\author{
Liliana Pedraja Rejas ${ }^{1}$ y Emilio Rodríguez-Ponce $e^{2,3}$
}

Los profesores poseen un papel fundamental en la sociedad del conocimiento, ya que son el primer eslabón en una cadena de formación permanente del capital humano de una nación. Por ende, sin profesores calificados no se puede generar capital humano avanzado de calidad y, por tanto, un país no puede ser competitivo (Di María y Lazarova, 2012; Mansoob y Serino, 2011).

La evidencia empírica muestra que en Chile las Facultades y/o Escuelas de Educación que operan en un entorno equivalente, con recursos y capacidades similares, exhiben diferentes niveles de calidad en su quehacer académico y en la calidad de sus egresados (Pedraja-Rejas, et al., 2012).

A partir de diversas investigaciones se puede observar la necesidad de relevar el rol de las Universidades, que cuentan con Facultades y Escuelas de Educación, a contribuir a formar profesores de primer nivel.

En este sentido uno de los aspectos fundamentales para generar una formación adecuada es contar con líderes que ejerzan un rol central en la formación universitaria y en la inspiración y amor por el trabajo a las nuevas generaciones.

El liderazgo es la influencia interpersonal, ejercida por un líder, mediante un proceso de comunicación para modificar las actitudes y el comportamiento de los seguidores, en orden a lograr objetivos organizacionales (Chuang et al., 2012; Northouse, 2010; Sung et al., 2010).

La mayoría de los trabajos académicos en el campo de la educación superior en los últimos años (por ejemplo, Ghorbani y Yekta, 2012; Mader, 2012; Richards, 2012); han tendido a concentrarse en la tipología de Bass y Avolio (1990), que clasifica y enfatiza en los estilos de liderazgo transformacional y transaccional.

El primero de los estilos mencionado, en el contexto del aula, se basa en el supuesto de que es posible concebir a esta como una pequeña organización social con el maestro como líder y los estudiantes como seguidores (Pounder, 2008). Bajo esta mirada, los seguidores van más allá de sus propios intereses y trabajan para el grupo. Así, es posible lograr mejoras en las organizaciones educativas, mediante un proceso de transformación de los seguidores, sus valores, sus actitudes, y sus motivaciones (Mawn et al., 2012; Tourish et al., 2010).

El carisma del líder afecta el desempeño mediante la interacción y el proceso de retroalimentación acerca de la tarea efectuada por los miembros de una facultad (Amin, 2012; Shah, 2009). Similarmente, existen investigaciones que han encontrado que el liderazgo transformacional está relacionado positivamente con la habilidad del líder para monitorear y manejar las emociones propias y de los seguidores (Shah, 2010, Sosik y Cameron, 2010). En consecuencia, en educación superior se ha comprobado la importancia del estilo de liderazgo transformacional para generar una visión compartida entre el líder y equipo, junto a compromiso, colaboración y una estimulación mutua, basada en el respeto por el líder, en su carisma, y en su capacidad para motivar a sus seguidores.

Corresponde entonces mencionar el caso del Departamento de Historia de la Universidad de Tarapacá donde el Profesor Luis Álvarez Miranda merece un homenaje puesto que fue un maestro de verdad, que contribuyó en la formación y generación

\footnotetext{
Universidad de Tarapacá, CEUTA, Arica, Chile. Correo electrónico: lpedraja@uta.cl

Universidad de Tarapacá, Arica, Chile. Correo electrónico: erodrigu@uta.cl

Los autores agradecen el patrocinio del CONICYT por medio del proyecto FONDECYT No 1140027 denominado: Estilos de liderazgo y calidad del quehacer académico en las Facultades y Escuelas de Educación de Chile.
} 
de competencias en los estudiantes e instalando una impronta en los profesores de la institución.

Se puede decir, por tanto que el Profesor Álvarez aportó decididamente en la formación de profesionales en el ámbito de la educación y definitivamente desde el ejercicio de su liderazgo transformador, el cual es ampliamente valorado en la literatura.
El presente número, en honor al Maestro Luis Álvarez Miranda, posee un conjunto de investigaciones que relevan la importancia de la interculturalidad y el estudio de la geografía, que desde el análisis morfométrico y la etnopercepción andina generan diversas aportaciones que contribuyen a la difusión de la investigación por medio de Diálogo Andino.

\section{Referencias Citadas}

Amin, M.

2012 The Relationship of Principals/Directors' Leadership Styles, as Perceived by the Faculty, to the Job Satisfaction of the Faculty Members in a Public University of Punjab, Pakistan Doctor of Education (Educational Leadership and Management) at the University of Leicester. https://lra.le.ac. uk/handle/2381/10774

Bass, B.

1990 From Transactional to Transformational Leadership: Learning to share the Vision.

Chuang, A., Judge, T., \& Jun, Y.

2012 Transformational leadership and customer service: A moderated mediation model of negative affectivity and emotion regulation. European Journal of Work and Organizational Psychology, 21(1), 28-56.

Di Maria, C., \& Lazarova, E. A.

2012 Migration, human capital formation, and growth: An empirical investigation. World Development, 40(5), 938-955.

Ghorbani, M., \& Yekta, S.

2012 A Study of Relationship Between Transformational Leadership and Personnel Creativity in Higher Education Centers. World Applied Sciences Journal 17(6), 684-693.

Mader, C.

2012 How to Assess Transformative Performance towards Sustainable Development in Higher Education Institutions, Journal of Education for Sustainable Development 6(1), 79-89.

Mansoob, S., \& Serino, L.

2011 The pattern of specialization and economic growth: The resource curse hypothesis revisited. Structural Change and Economic Dynamics, 22(2), 151-161.

Northouse, $\mathrm{P}$.

2010 Leadership: Theory and practice (5a. Ed.). Thousand Oaks, CA: Sage.
Mawn, L., Callow, N., Hardy, J., \& Arthur, C.

2012 Development and Assessment of a Transformational Leadership Inventory in Higher Education Final Report Bangor University.

Pedraja Rejas, L.

2012 Desafíos para el profesorado en la sociedad del conocimiento. Ingeniare. Revista chilena de ingeniería, 20(1), 136-144.

Richards, D.

2012 Leadership for Learning in Higher Education: The Student Perspective.

Pounder, J.

2008 Transformational classroom leadership: a novel approach to evaluating classroom performance, Assessment \& evaluation in higher education, 33(3), 233-243.

Shah, S.

2010 Re-thinking Educational Leadership: Exploring the Impact of Cultural and Belief Systems. International Journal of Leadership in Education, 13(1), 27-44.

Shah, $\mathrm{S}$.

2009 The Impact of Societal Culture on Practice: People Management in Colleges in Pakistan. Journal of Educational Leadership, Policy and Practice, 24(2), 3-17.

Sosik, J. J., \& Cameron, J. C.

2010 Character and authentic transformational leadership behavior: Expanding the ascetic self towards others. Consulting Psychology Journal: Practice and Research, 62(4), 251-269.

Sung, C., Chia-Hui, L., Chen, Y., \& Chen Y.

2010 Leadership Styles and Teachers' Job Satisfaction in Taiwan, Higher Education. http://research.toko.edu.tw/ ezfiles/9/1009/attach/19/pta_6299_4367587_36283.pdf

Tourish, D., Craig, R., \& Amernic, J.

2010 Transformational Leadership Education and Agency Perspectives in Business School Pedagogy: A Marriage of Inconvenience? British Journal of Management, 21, 40-59. 